\title{
High-frequency collective excitations in molten and glassy Te studied by inelastic neutron scattering
}

\author{
M. D. Ruiz-Martín, ${ }^{1}$ M. Jiménez-Ruiz, ${ }^{1}$ F. J. Bermejo, ${ }^{2}$ and R. Fernández-Perea ${ }^{3}$ \\ ${ }^{1}$ Institut Laue Langevin, 6 Rue Jules Horowitz, BP. 156, F-38042 Grenoble Cedex 9, France \\ ${ }^{2}$ C.S.I.C., Department of Electricity and Electronics, UPV/EHU, Box 644, 4880 Bilbao, Spain \\ ${ }^{3}$ Instituto de Estructura de la Materia, Consejo Superior de Investigacioens Científcas, Serrano 123, E-28006 Madrid, Spain
}

(Received 6 December 2005; revised manuscript received 3 February 2006; published 20 March 2006)

\begin{abstract}
The spectra of collective excitations of liquid and glassy tellurium have been studied by means of inelastic neutron scattering. Here we report on the dynamics of liquid Te as measured at two different temperatures, just above melting $\left(T_{m}=723 \mathrm{~K}\right)$ and at $\sim 1000 \mathrm{~K}$ as well as the glass that is studied at room temperature. Estimates for the velocity of propagating excitations for both temperatures have been obtained from the experimental data, and a contrasting behavior is found with respect to anomalies shown by the adiabatic sound velocity measured by ultrasound methods. The origin of such differences is finally discussed.
\end{abstract}

DOI: 10.1103/PhysRevB.73.094201

PACS number(s): 61.25.Mv, 61.43.Er, 61.20.Lc, 61.12.-q

\section{INTRODUCTION}

Condensed tellurium constitutes an exceedingly interesting material from the point of view of its physical properties. Crystalline Te at ambient pressure is a trigonal, twofoldcoordinated semiconductor having three atoms per unit cell arranged helically along the crystal $c$ axis. Bonding within the helical chains is known to be covalent while interchain interactions are mostly van der Waals. It transforms into a relatively poor metallic conductor upon melting at $T_{m}$ $=723 \mathrm{~K}^{1}{ }^{1}$ The transport and optical properties of liquid tellurium ( $l-\mathrm{Te})$ reveal far more complex behaviors than any other liquid metal or semiconductor ${ }^{1-4}$ that is known to arise from changes in the bonding structure. In fact, the electrical resistivity decreases upon melting by about $10 \%$ down to a value of $\sim 500(\mu \Omega \mathrm{cm})^{-1}$ and shows a mild temperature variation, reaching $\sim 375(\mu \Omega \mathrm{cm})^{-1}$ at $1250 \mathrm{~K}$.

Some other basic properties such as the liquid density also exhibit noticeable anomalies such as the presence of a clear maximum at $T_{m}$; that is, the liquid density decreases as the temperature is lowered into the supercooled liquid range. ${ }^{4}$

Several details of the microscopic bonding structure within the liquid have been under discussion since the pioneering work of Cabane and Friedel, ${ }^{5}$ who proposed a model of a threefold-coordinated random network. Our current view $^{6}$ on the structure and bonding of $l$-Te portrays some survival within the liquid of the chain structure of the crystal with significant changes in bond length and bond angles. The coordination number increases with temperature from roughly 2 at melting up to about 2.8 at $1100 \mathrm{~K}$ as it also does with applied pressures of $4 \mathrm{GPa}^{6}{ }^{6}$ Such an increase in coordination number arises from the breakdown of the structure of the interchain neighbor shell upon melting and correlates with a noticeable change in electronic structure which chiefly affects the lone-pair band which broadens upon melting while the density of states at the Fermi energy still retains a nonvanishing value. ${ }^{7,8}$

The temperature dependence of the macroscopic sound velocity of $l$-Te is perhaps the most tantalizing property of the liquid. Early reports ${ }^{9}$ showed a value for the adiabatic sound velocity that, contrary to what has been registered for most liquids, is increased from some $800 \mathrm{~m} \mathrm{~s}^{-1}$ at melting up to about $1150 \mathrm{~m} \mathrm{~s}^{-1}$ at $1100 \mathrm{~K}$. Such an unusual dependence was confirmed by later studies ${ }^{10}$ and attributed to the structural changes accompanying the increase in coordination number. The phenomenon seems to correlate with the presence within the liquid of a relatively large number of conduction electrons.

Here we report on inelastic neutron scattering studies in $l$-Te measured at temperatures where the sound velocity still increases upon heating $(773 \mathrm{~K})$ as well as at $1023 \mathrm{~K}$ where the temperature dependence of the sound velocity becomes far milder. The rationale behind our study is twofold. First we aim to explore whether the macroscopic finding on the anomalous behavior of the sound velocity also has a microscopic correlate in the excitation frequencies as measured within the microscopic realm and thus help to establish a direct connection between changes in bonding structure and the elastic properties of the medium. Second, and on a more fundamental vein, this material can be taken as a case point concerning ensuing discussions of the propagation of excitations in disordered media. More into specifics, the focus of our attention concerns some highly polarized features appearing within the frequency range $6-16 \mathrm{meV}$ in the Raman spectra $^{11}$ which are highly a typical for a liquid, since there one would rather expect to find broad, depolarized features. Its origin is believed to be related to the presence even within the liquid of $A$-like modes, usually assigned to chain rotation and expansion, ${ }^{12}$ which are tightly coupled to the electron system and therefore the motion of ions within such modes leads to substantial rearrangements of the electron cloud. This conjecture is also given further support from studies carried out in small Te clusters ${ }^{13}$ by means of Raman and photoelectron spectroscopies which also report on modes of such symmetry vibrating at frequencies of several meV. Under these circumstances it is thus of interest to explore whether the presence within the liquid of the modes just referred to affects the excitations measured within the liquid at wave vectors which would correspond to those well within the first Brillouin zone of the parent crystal and usually are 
interpreted in terms of the propagation of some acousticlike mode.

Although several reports on neutron inelastic scattering measurements on the liquid have already appeared in the literature, ${ }^{14-16}$ their main focus has been the determination of quasielastic scattering widths as well as rough estimates for the spectral frequency distributions. In what follows we will describe our measurements for the liquid sample performed on a thermal triple-axis neutron spectrometer as well as some measurements for a glassy sample that are here taken as a reference.

\section{EXPERIMENTAL DETAILS}

The sample (99.999\% purity) was held within a quartz container of $12 \mathrm{~mm}$ of outer diameter and $1.2 \mathrm{~mm}$ of thickness. These dimensions were calculated in order to have $\sim 20 \%$ the incoming neutrons scattered and/or absorbed by the sample account made of the neutron scattering and absorption cross sections of the studied element, $\sigma_{s}=4.32 \mathrm{~b}$ and $\sigma_{a b s}=4.7 \mathrm{~b}$, respectively.

Two different thermodynamic states have been studied within the liquid and one within the glass phase. The liquid was obtained by heating the sample up to its the melting point $T_{m}=723 \mathrm{~K}$ inside a niobium furnace. In order to create the vitreous state, a rapid quench from the liquid into dry ice was performed. Because of the need of a bulk sample, we had to follow a different route from that previously employed in optical spectroscopy ${ }^{8}$ which yields micron-thick films by vapor deposition onto a substrate held at about $150 \mathrm{~K}$. For such a purpose, the sample was heated up to $873 \mathrm{~K}$ for $2 \mathrm{~h}$ inside a furnace and then quickly immersed into dry ice at a temperature of $\sim-80{ }^{\circ} \mathrm{C}$, which ensures a fast crossing through the glass transition temperature $\left[T_{g}=343 \mathrm{~K}\right.$ (Ref. 17)].

The experiment was carried out on the recently rebuilt thermal three-axis spectrometer IN8c, at the Institut Laue Langevin (ILL). The optimal conditions yielding maximum flux and relatively high resolution in energy transfers were found to be those employing a $\mathrm{Cu}(200)$ monochromator and $\mathrm{a} \mathrm{Cu}(111)$ analyzer, using horizontal collimations of $15^{\prime}-30^{\prime}-20^{\prime}-20^{\prime}$. For the liquid, the experiment was carried out in constant final wave-vector mode with $k_{f}=4.1 \AA^{-1}$. The energy resolution as measured at the elastic peak position using vanadium foil for this configuration was a Gaussian of $0.9 \mathrm{meV}$, full width at half maximum (FWHM). Since the sound velocity of liquid tellurium is of the order of $1000 \mathrm{~m} \mathrm{~s}^{-1}$, all the excitations could be within reach when working at this $k_{f}$. For the glass phase, since the excitations were expected to appear at higher energies, higher $k_{f}$ values were required and therefore the final wave vector had to be increased to $k_{f}=5.5 \AA^{-1}$ which yielded a resolution of $2.2 \mathrm{meV}$ at the elastic line as measured with a vanadium rod of diameter $\sim 11 \mathrm{~mm}$.

The sample was placed inside a vacuum box, where the air pressure was lower than $10^{-4} \mathrm{mbar}$, in order to prevent scattering from air. The sample container was heated up to $773 \mathrm{~K}$, and then, several elastic and inelastic scans were performed. The elastic scan was made for wave vectors
0.1-3.0 $\AA^{-1}$ and the inelastic scans for wave vectors $0.3-0.7 \AA^{-1}$. Under our experimental conditions, scattering from the sample container was found to yield virtually pure elastic signals; that is, scattering from the walls of the container was too weak to be detected.

The sample was heated up to $\sim 723 \mathrm{~K}$ at a rate of $450{ }^{\circ} \mathrm{C} / \mathrm{h}$. Melting was monitored as Bragg peaks from crystalline Te disappeared from elastic scans which yield a rough estimate of the static structure factor $S(Q)$. When melting was achieved a broad liquid peak appeared at $Q \simeq 2 \AA^{-1}$, which corresponds to the first diffraction peak of the liquid as reported several times. ${ }^{18}$

Several scans at constant $Q$ versus energy transfer were carried out from $0.3 \AA^{-1}$ up to $0.7 \AA^{-1}$ for two different temperatures $773 \mathrm{~K}$ and $1023 \mathrm{~K}$. As was referred to above both values were chosen since the first comes close to melting and the sound velocity of $l$-Te at the latter temperature reaches its maximum. At higher temperatures, it remains almost constant. Thus, we could explore two regions of the melt, which may display different properties. We collected data up to wave-vector transfer values of $0.7 \AA^{-1}$, since above this $Q$ value, the excitations were overdamped and therefore very scarce information was obtainable from wave vectors beyond that value.

Because of the relatively low intensities and the low scattering angles, special precautions had to be taken to subtract all those backgrounds arising from different sources such as the inherent spectrometer background noise, instrumental artifacts, and scattering from sample environment devices. The spectrometer background noise was found to be $\sim 7$ counts/min at an energy transfer of $\sim 4 \mathrm{meV}$.

At the low angles required for studying the collective dynamics in a liquid, scattering from the tails of the direct beam has to be handled with care, using for this purpose measurements using a Cd mockup.

Finally, the last correction to be applied to the measured intensities involved the estimation of the multiply scattered neutrons. For such a purpose computer simulations were carried out using a modified version of the MSCAT code. ${ }^{19}$ As an initial scattering kernel we have used an estimate for the quasielastic part of the spectra calculated from reported values of the self-diffusion coefficient ${ }^{15}$ and using the Sköld approximation. The results turned out to be not very sensitive to the choice of scattering kernel, mostly because of the relatively large neutron-capture cross section of Te which is comparable to the total scattering cross section $\left(\sigma_{a b s} / \sigma_{\text {scatt }}\right.$ 1.09). An example of what this contribution amounts to is given below.

\section{RESULTS}

After performing the monitor normalization and the cadmium subtraction, the measured data $I(Q, \omega)$ defined as

$$
I(Q, \omega) \propto S(Q, \omega) \otimes \mathfrak{R}(Q, \omega)+N_{\mathrm{bcgr}},
$$

where $\mathfrak{R}(Q, \omega)$ is the spectrometer resolution function given by 


$$
\mathfrak{R}(Q, \omega)=\frac{1}{\sqrt{2 \pi \sigma^{2}}} \exp \left(-\frac{\omega^{2}}{2 \sigma^{2}}\right),
$$

with $\sigma^{2}$ the standard Gaussian deviation. Both the value of such a parameter and the shape of the instrument function were calibrated against the measurements carried out using a vanadium rod having a geometry matching that of our sample. $N_{\text {bcgr }}$ is a background term that is left as adjustable. The quantity $S(Q, \omega)$ encompasses all the information on the liquid dynamics, and as a workable approximation we have modeled it in terms of a quasi elastic as well as a purely inelastic component,

$$
S(Q, \omega)=S_{q e l}(Q, \omega)+S_{\text {inel }}(Q, \omega),
$$

where $S_{q e l}(Q, \omega)$ stands for the central (quasielastic) part of the studied spectrum and $S_{\text {inel }}(Q, \omega)$ refers to the part containing information about collective dynamics. The quasielastic part, which provides information about the stochastic processes, which are all those whose average frequency is zero, is modeled with a Lorentzian function,

$$
S(Q, \omega)_{\text {quasi }}=\frac{1}{\pi} \frac{\Delta_{\omega}}{\omega^{2}+\left(\Delta_{\omega}\right)^{2}},
$$

where $\Delta_{\omega}$ is the half width at half maximum (HWHM) of the peak. In turn, the inelastic part is modeled in terms of a damped harmonic oscillator,

$$
S(Q, \omega)_{i n e l}=H(Q) \omega[n(\omega)+1] \frac{4 \omega \Gamma}{\left(\omega^{2}-\Omega^{2}\right)^{2}+4 \omega^{2} \Gamma^{2}},
$$

formulated in terms of a renormalized excitation frequency $\Omega$, given by

$$
\Omega^{2}=\omega^{2}+\Gamma^{2},
$$

where $\omega$ is the bare oscillator frequency and $\Gamma$ is the damping, and both quantities are assumed to show a significant wave-vector dependence. $H(Q)$ is an amplitude coefficient and $n(\omega+1)$ is the Bose occupation factor, defined as $1 /\left(e^{\hbar \omega / K_{B} T}-1\right)$. As far as the background term $N_{\text {bcgr }}$ it shows a non-negligible slope due to the high-absorption crosssection value which makes it to be energy dependent since

$$
\sigma_{a b s}=\sigma_{a b s}^{0} \sqrt{\frac{E_{0}}{E}}
$$

where $\sigma_{a b s}^{0}$ stands for the value of the neutron-absorption cross section of the studied element at $E_{0}=25 \mathrm{meV}$. This makes higher-energy neutrons to be less absorbed than those having a lower energy and thus results in a measurable slope within the background that is accountable using a linear term.

\section{A. Liquid tellurium}

A set of corrected and fitted spectra is displayed in Fig. 1, where the excitations that appear in both stokes and antiStokes sides of the spectra can be seen clearly.

As expected at the low wave vectors explored most of the quasielastic scattering will here arise from the small but mea-

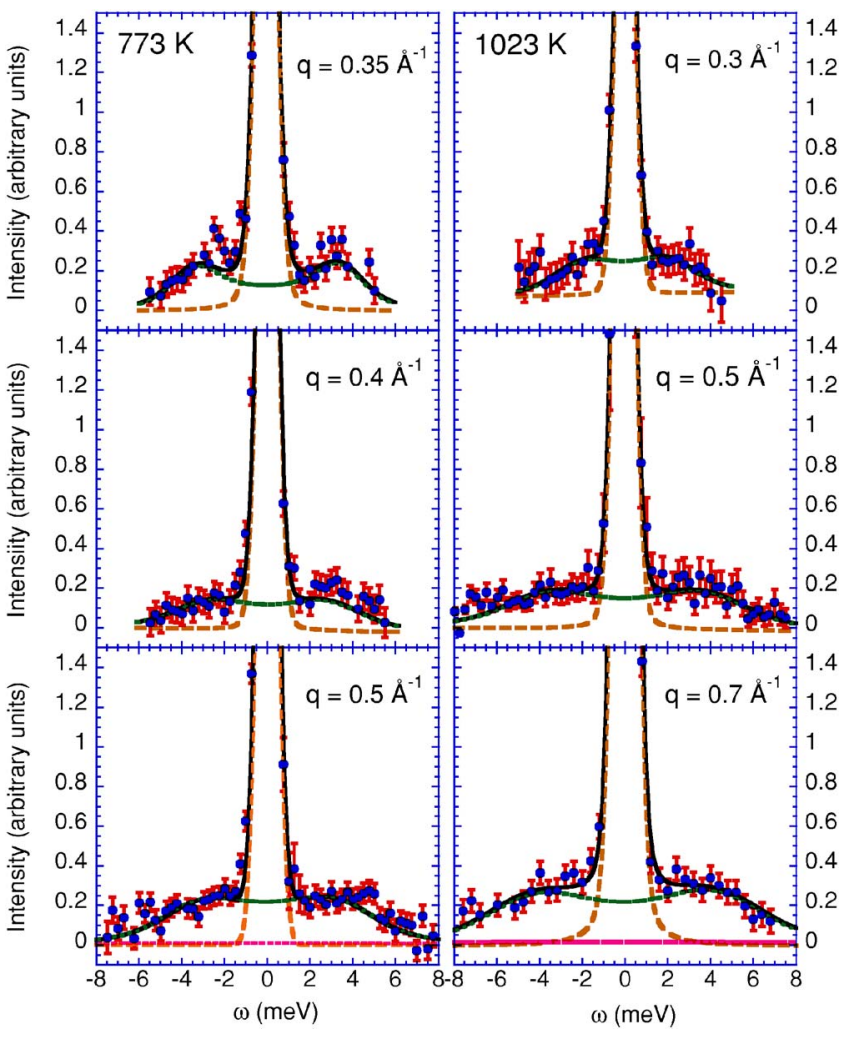

FIG. 1. (Color online) Several fitted spectra for liquid tellurium at $773 \mathrm{~K}$ and $1023 \mathrm{~K}$. Experimental data are shown by solid symbols. The model intensities $I(Q, \omega)$ are depicted by the solid line. Its Lorentzian component centered at the elastic line is shown by the dashed line. The damped harmonic oscillator that takes into account the excitations that appear at the finite frequencies is shown by the dotted line. The lower two frames also depict the contribution due to multiple scattering that has a $($ FWHM $\sim 38 \mathrm{meV})$.

surable incoherent cross section of Te $(0.1 \mathrm{~b})$ plus some possible coherent quasielastic contribution having a linewidth and a $Q$ dependence that cannot be a priori predicted. The derived linewidths were found to be close to those already reported by other studies ${ }^{14,15}$ and show a deceptively simple wave-vector dependence that is $\Delta_{\omega}=2 D_{s} Q^{2}$ where $D_{s}$ stands for the self-diffusion coefficient. This trend is displayed in Fig. 2. The results for the quasi elastic part of the spectrum are found to be in agreement with those reported by Axmann et al. ${ }^{15}$ measured using cold-neutron spectroscopy and a significantly higher resolution in energy transfers than ours as well as those reported by Chiba et al. ${ }^{20}$ measured using a lower resolution but extending up to far larger wave vectors. In both cases it is found that below $\approx 1 \AA^{-1}$ the quasielastic linewidth follows the Fick law using previously published values $^{15}$ for the self-diffusion coefficient. Our own estimate for $D_{s}=2.28 \times 10^{-5} \mathrm{~cm}^{2} \mathrm{~s}^{-1}$ compares favorably to that of $D_{s}=2.6 \times 10^{-5} \mathrm{~cm}^{2} \mathrm{~s}^{-1}$ reported in a previous study employing a far higher resolution in energy transfers $[0.27 \mathrm{meV}$ (Ref. 15)] and compares to an estimate of the macroscopic value for the same quantity at $T=743 \mathrm{~K}$ of $D_{s}=2.9$ $\times 10^{-5} \mathrm{~cm}^{2} \mathrm{~s}^{-1} \cdot 4,15$

The result is somewhat surprising account made of the ratio of incoherent to coherent neutron cross sections 


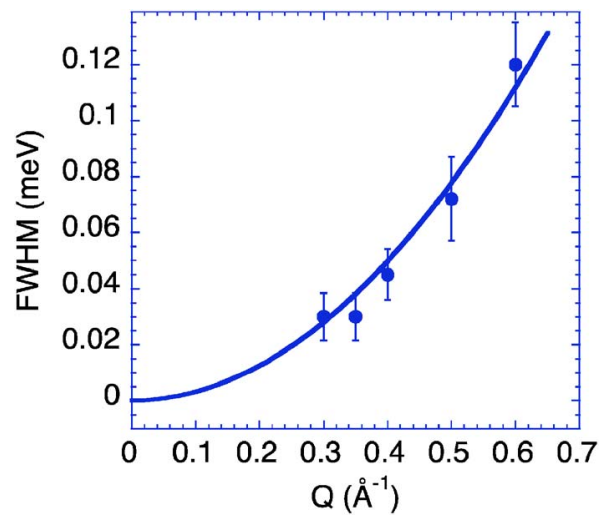

FIG. 2. (Color online) $Q$ dependence of the linewidth of $l$-Te at $773 \mathrm{~K}$. The same kind of dependence was found for the hightemperature melt. The solid line represents a quadratic dependence with wave vector and yields a value for the apparent self-diffusion coefficient $D_{s}=2.28 \times 10^{-5} \mathrm{~cm}^{2} \mathrm{~s}^{-1}$.

$\sigma_{\text {incoh }} / \sigma_{\text {coh }}=0.023$ and suggests that both quasielastic components do have comparable linewidths and wave-vector dependence. This is known to be the case for molten simple alkali metals such as $\mathrm{K},{ }^{21}$ but goes counter to what is found for complex liquids such as $\mathrm{Ga}^{22}$

The wave-vector dependence of the parameters obtained after fitting the experimental results with Eq. (3), $\omega$ and $\Gamma$, is shown in Fig. 3. From there, an estimate of the excitation velocity and the damping coefficient can be obtained, using linear and power fits with respect to the wave vector, respectively.

The values obtained for the asymptotic value of the excitation velocity yielding an estimate for the sound velocity and the damping coefficient for both temperatures are given in Table I. From there we see that the differences in slope of $\Omega$ for the two temperatures in question are comparable (i.e., within error bars). In contrast, a clearer difference is found concerning the damping terms. These show a nonhydrodynamic dependence, departing from the $\Gamma \propto Q^{2}$ expected to be obeyed at long wavelengths, and are better approximated by a generalization yielding $\Gamma \propto Q^{\delta}$, where the exponent value seems to be dependent upon the thermodynamic state.

With the proviso in mind of the paucity of data from the present results it is clear that the parameters characterizing the excitations being sampled at our meV frequencies do not follow the same trends exhibited at macroscopic scales. ${ }^{9,10}$ The results can be compared to those arising from a recent calculation $^{23}$ on $\mathrm{Te}_{1-x} \mathrm{I}_{x}$ based upon empirical potentials derived from the static structure factors. In the limit of low iodine concentration the value calculated as an extrapolation to $x-\rightarrow 0$ yields $1700 \mathrm{~m} \mathrm{~s}^{-1}$.

\section{B. Glassy tellurium}

A similar fitting procedure as for the liquid phase was performed for $g$-Te, taking into account in this case that the quasielastic contribution should be negligible, due to the fact that our sample is a solid and any low-frequency motions characteristic of the glassy state are undetectable in our fre-

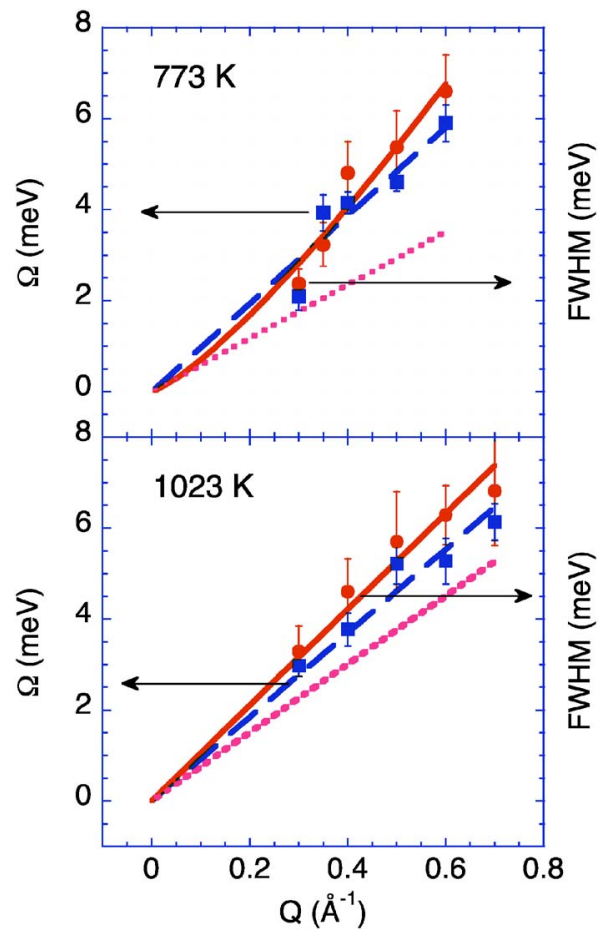

FIG. 3. (Color online) Dispersion curve for $l$-Te for both temperatures $773 \mathrm{~K}$ and $1023 \mathrm{~K}$. Solid squares represent the frequency of the phonon excitations, and the full widths at half maximum of the peaks are represented by the solid circles. The dashed line shows the hydrodynamic linear dispersion calculated from values for the sound velocity derived from ultrasound measurements. The two solid lines show fits to the wave-vector dependences described in the text.

quency window. The fitted experimental data are shown in Fig. 4.

As for the liquid results, the sound velocity and the damping coefficient were obtained from the fitting parameters of the curves shown in Fig. 5, which display the $Q$ dependence of the oscillator frequency and damping. The derived values for the relevant parameters are given in Table I.

\section{DISCUSSION}

The most remarkable result from the present study concerns the rather disparate variation in the temperature depen-

TABLE I. Experimental estimates for the excitation velocities $v_{s}^{\text {expt }} \lim _{Q \rightarrow 0} \Omega / Q$ and their corresponding macroscopic values $v_{s}$. Parameter values for excitation damping $\Gamma=D Q^{\delta}$ as obtained from the present study.

\begin{tabular}{lcccc}
\hline \hline Liquid & $v_{s}^{\text {expt }}\left(\mathrm{m} \mathrm{s}^{-1}\right)$ & $v_{s}^{s}\left(\mathrm{~m} \mathrm{~s}^{-1}\right)$ & $D\left(\mathrm{meV}^{\delta}\right)$ & $\delta$ \\
\hline $773 \mathrm{~K}$ & $1470 \pm 80$ & 890 & $12.9 \pm 2.1$ & $1.26 \pm 0.2$ \\
$1023 \mathrm{~K}$ & $1400 \pm 50$ & 1140 & $10.54 \pm 0.35$ & $1.0 \pm 0.2$ \\
Glassy & $v_{s}^{\text {expt }}\left(\mathrm{m} \mathrm{s}^{-1}\right)$ & $v_{s}^{\text {th }}\left(\mathrm{m} \mathrm{s}^{-1}\right)$ & $D\left(\mathrm{meV}^{\delta}\right)$ & $\delta$ \\
\hline $293 \mathrm{~K}$ & $2298 \pm 60$ & & $25.8 \pm 3.6$ & $2.30 \pm 0.35$ \\
\hline \hline
\end{tabular}




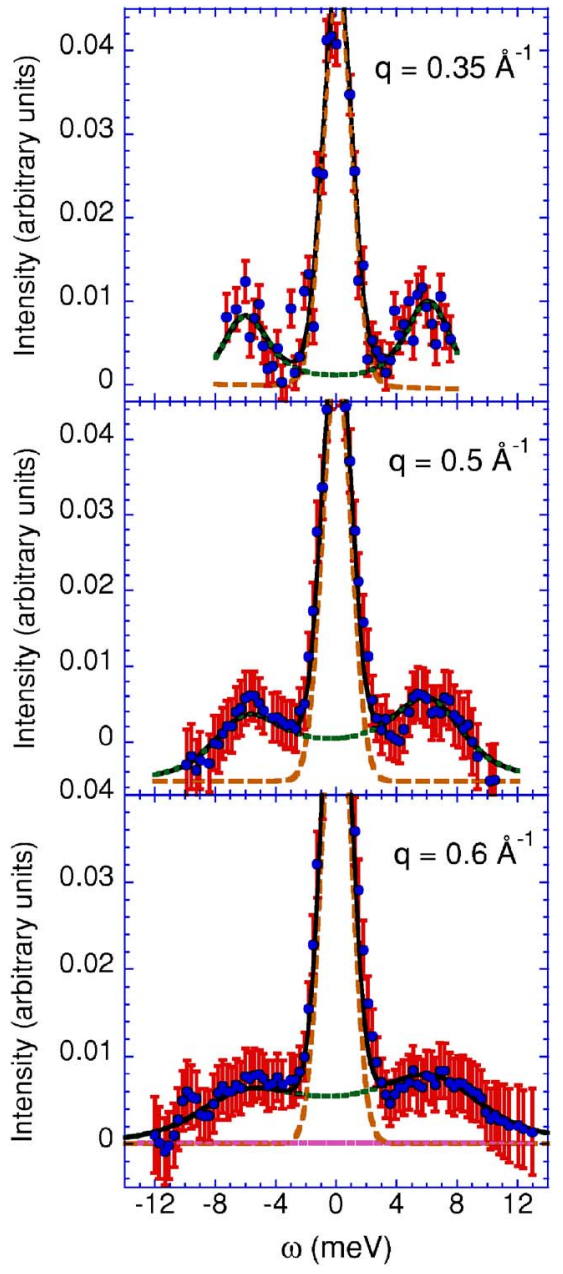

FIG. 4. (Color online) Several fitted spectra for glassy tellurium at room temperature. Solid symbols show fully corrected experimental data, and the model $I(Q, \omega)$ is shown by the solid line. The elastic line is shown by the dashed (brown) line, and the damped harmonic oscillator (DHO) is shown by the dotted (green) line. The two solid lines show fits to the wave-vector dependences described in the text.

dence of the sound velocities derived from mechanical spectroscopy 9,10 and those from the present measurements. Our estimates for the liquid lay well above those found in the study using macroscopic means. Such a large difference cannot be attributed to the different thermodynamics being explored by both techniques. As a matter of fact, estimates for the ratio of specific heats $\gamma=C_{p} / C_{v}$ using for the purpose data reported in Ref. 10 for thermal expansion and adiabatic compressibility as well as tabulated data ${ }^{3}$ for the molar heat capacity yield values for $\gamma=1.04$ at melting, and therefore the differences between isothermal and adiabatic sound velocities lie within the experimental error.

In principle, measurements carried out within the kinematic range accessible to neutron scattering sample the highfrequency sound velocity $c_{\infty}=\sqrt{3 k_{B} T / M+3 \omega_{E}^{2} R_{0}^{2} / 10}$ where $\omega_{E}$ stands for a characteristic Einstein frequency and $R_{0}$ is an atomic diameter with a value set to that corresponding to the main minimum of the atomic interaction potential. Setting $R_{0}=2.86 \AA$, which corresponds to the most frequently used

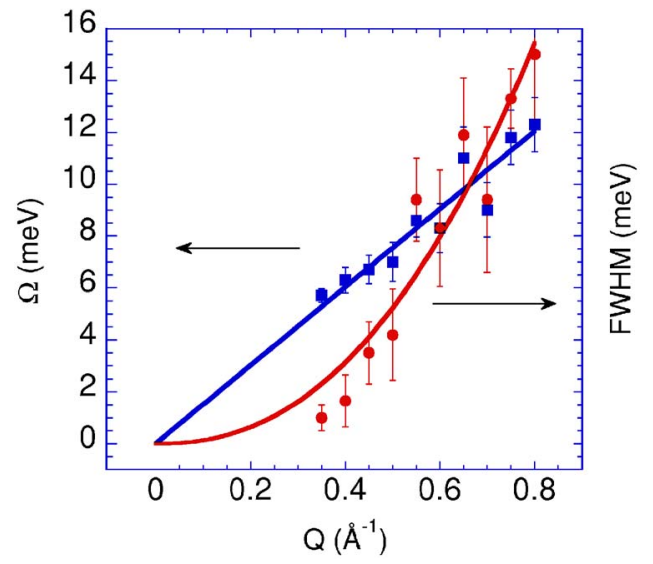

FIG. 5. (Color online) Excitation frequencies and linewidths for glassy tellurium studied with $k_{f}=5.5 \AA^{-1}$. Solid circles depict the excitation linewidths $\Gamma$, and squares show the renormalized frequencies.

value in tight-binding calculations, ${ }^{6}$ and equating $c_{\infty}$ to the values herein measured one gets an estimate of the Einstein frequency $\omega_{E}=6.12 \mathrm{meV}$ at $\mathrm{T}=773 \mathrm{~K}$. The figure comes close to the maximum of estimates for the spectral frequency distributions reported on by Chiba et al. ${ }^{24} \mathrm{~A}$ similar calculation using the higher-temperature data yields a close value (5.81 meV).

The data herein presented showing a distinct dependence of the temperature dependence of the high-frequency sound velocity from that measured ultrasonically provide a vivid reminder that the response of a liquid sample to a highfrequency probe such are thermal neutrons is better understood in terms of that corresponding to the instantaneous response of an elastic medium where the velocity of the collective oscillation is given by $\sqrt{\left(B+\frac{4}{3} G\right) / M \rho}$ in terms of the bulk $(B)$ and rigidity $(G)$ moduli and $M \rho$ stands for the mass density than to that predicted from linearized hydrodynamic equations that yield $\left(\sqrt{\chi_{s} M \rho}\right)^{-1} \cdot{ }^{25}$ By construction, the velocity sampled by a high-frequency probe will always be equal or higher than the adiabatic value given by the adiabatic compressibility $\chi_{s}$ that is actually measured by ultrasonic experiments. The same consideration applies to data for the glass as well as already reported data for glassy $\mathrm{Se},{ }^{26}$ which again shows a noticeable deviation from hydrodynamics.

The data derived concerning $\omega_{E}$ match the lower bound for the frequency of the $A$ modes found in Raman spectra of the liquid as well as from studies of small atomic clusters. ${ }^{13}$ It may be related to microscopic details given by the pair correlation function $g(r)$ and the interatomic potential $V(r)$ through the integral, ${ }^{25}$

$$
\omega_{E}^{2}=\frac{4 \pi \rho}{3 M} \int_{0}^{\infty} r^{2} d r g(r) V^{\prime \prime}(r),
$$

where the double prime stands for the second derivative. The received wisdom tells that $\omega_{E}$ should be close to the maximum frequency of the crystalline solid and, in fact, from single-crystal phonon dispersion relations ${ }^{12}$ it is seen that it 
roughly corresponds to the upper bound for the acoustic branches in trigonal $\mathrm{Te}$.

Consideration of the wave-vector and temperature dependence of the excitation linewidths provides additional support for the view here put forward. With the proviso in mind of the small number of data points available for the liquid, our results show that the high-temperature melt is better fitted by a power law $\Gamma(Q) \propto Q^{\delta}$ with the exponent $\delta \simeq 1.0$ than by a quadratic wave-vector dependence, mimicking the hydrodynamic result. A similar exercise carried out for the liquid close to melting yields a higher value for $\delta$ while that for the glass gives $\delta=2.3$. In other words, if interpreted in terms of scattering of sound waves, the result shows that the precise wave-vector dependence followed by the damping coefficient is dependent upon the thermodynamic state being explored. The result thus provides a warning against some proposed generalizations to account for the wave-vector dependence of the spectral linewidth. ${ }^{26,27}$

The data concerning the liquid show that the damping of collective oscillations at a given value of the wave vector $\mathrm{Q}=0.4 \AA^{-1}$ decreases by mere $6 \%$ upon rising temperature. In stark contrast, the shear viscosity which is thought to be the main contributor to damping within the hydrodynamic realm shows a threefold decrease within the explored range of temperature. ${ }^{4,28}$ Alternatively, one may interpret the observed linewidths as a signature of excitation lifetimes as $t$ $=\pi / \Gamma$. On such grounds one gets excitation lifetimes close to melting of about $0.69 \mathrm{ps}$ for $Q=0.4 \AA^{-1}$. This, taken together with the estimate for the high-frequency sound velocity shows that the excitations being sampled may travel significantly long distances $(l=10 \AA)$. Notice that the estimate for the lifetime of the excitation comes significantly shorter than the figure of $\tau=6 \mathrm{ps}$ which was inferred from the relationship $\tau \simeq l_{j}^{2} / 6 D_{s}$, where $l_{j}$ stands for a jump distance, and it has been identified with the lifetime of an interatomic bond within the liquid. ${ }^{15}$

Data measured for the glass are here taken as a reference. The results show, as expected, a significant increase in exci- tation frequencies concomitant with a far less important increase in excitation linewidths. Our results can be directly compared to those measured for glassy Se by means of inelastic scattering of $\mathrm{x}$ rays. ${ }^{26}$ In fact our results for the sound velocity and damping terms compare to those of $2000 \mathrm{~ms}^{-1}$ and $\approx 15 \mathrm{mev} \AA^{2}$ reported for glassy Se as measured at $\mathrm{T}$ $=295 \mathrm{~K}$. Assuming that the interparticle interactions at short range are not dramatically altered upon melting one can relate the excitation velocity of the two materials taking as a reference their trigonal crystalline ground states ${ }^{12}$ for which a complete set of elastic-constant data is available. The elastic and rigidity moduli can then be computed after an isotropic averaging of the $c_{11}, c_{44}$, and $c_{12}$ elastic constants is carried out following established procedures. ${ }^{29}$ From there one obtains a ratio of $c_{\infty}$ for the two materials, $c_{\infty}^{T e} / c_{\infty}^{S e}=1.17$, which compares to that of 1.15 evaluated from present and previous ${ }^{26}$ data.

In summary, our results for liquid $\mathrm{Te}$ - that it is a reactive liquid where interatomic bonds have lifetimes within the picosecond range- show that its microscopic dynamics at low wave vectors and frequencies can still be understood in terms of concepts developed for simple liquids. The effects of directional interactions such as those involved in tear and repair of covalent bonds are comprised within some of the model parameters such as the large value found for the excitation phase velocities if compared to those derived from macroscopic measurements as well as the associated Einstein frequency.

\section{ACKNOWLEDGEMENTS}

This work was supported in part by Grant No. MAT200204178-C04 (Spain). The authors wish to thank P. Palleau and S. Baudoin from the I.L.L. (Grenoble) for excellent technical support with the measurements. The authors wish to thank P. Martin and S. Demas for help given with sample environment issues.
${ }^{1}$ J. C. Perron, Adv. Phys. 16, 657 (1967); B. Cabane and C. Froidevaux, Phys. Lett. 29A, 512 (1969).

${ }^{2}$ J. N. Hodgson, Philos. Mag. 3, 735 (1963); D. A. Barlow, Phys. Rev. B 69, 193201 (2004).

${ }^{3}$ T. IIda and R. I. L. Guthrie, The Physical Properties of Liquid Metals (Oxford Science, Oxford, 1998), Chap. 8, p. 226.

${ }^{4}$ C. Li, C.-H. Su, S. L. Lehoczky, R. N. Scripa, B. Lin, and H. Ban, J. Appl. Phys. 97, 083513 (2005).

${ }^{5}$ B. Cabane and J. Friedel, J. Phys. (Paris) 32, 73 (1971).

${ }^{6}$ C. Bichara, J.-Y. Raty, and J.-P. Gaspard, Phys. Rev. B 53, 206 (1996); N. Funamori and K. Tsuji, ibid. 65, 014105 (2001); J. E. Enderby and A. C. Barnes, Rep. Prog. Phys. 53, 85 (1990).

${ }^{7}$ M. G. Garnier, E. Wahrenverg, and P. Oelhafen, Phys. Rev. B 65 , 113204 (2002).

${ }^{8}$ N. J. Shevchik, M. Cardona, and J. Tejeda, Phys. Rev. B 8, 2833 (1973); J. D. Joannopoulos, M. Schlüter, and M. L. Cohen, ibid. 11, 2186 (1975); L. D. Laude, R. F. Willis, and B. Fitton, Phys. Rev. Lett. 29, 472 (1972).
${ }^{9}$ M. B. Gitis and I. G. Mikhailov, Sov. Phys. JETP 12, 14 (1966).

${ }^{10}$ K-J. Sing and Y. Tsuchiya, Eur. Phys. J. B 12, 235 (1995).

${ }^{11}$ J. R. Magaña and J. S. Lannin, Phys. Rev. Lett. 51, 2398 (1983).

${ }^{12}$ R. M. Martin, G. Lucovski, and K. Helliwell, Phys. Rev. B 13, 1383 (1976).

${ }^{13}$ J. Becker, K. Rademann, and F. Hensel, Z. Phys. D: At., Mol. Clusters 19, 233 (1991); V. V. Poborchii, Chem. Phys. Lett. 251, 230 (1996). For a density functional theory calculation on clusters of varying sizes see B. C. Pan, Phys. Rev. B 65, 085407 (2002); 59, 3501 (1999).

${ }^{14}$ H. Endo, T. Tsuzuki, M. Yao, Y. Kawakita, K. Shibata, T. Kamiyama, M. Misawa, and K. Suzuki, J. Phys. Soc. Jpn. 63, 3200 (1994).

${ }^{15}$ A. Axmann, W. Gissler, A. Kollmar, and T. Springer, Discuss. Faraday Soc. 50, 74 (1970).

${ }^{16} \mathrm{~W}$. Gissler, A. Axmann, and T. Springer, in Proceedings of the Conference on Inelastic Scattering of Neutrons, edited by International Atomic Energy Agency, Vienna (IAEA, Vienna, 1968), 
Vol. 1, p. 245.

${ }^{17}$ D. J. Sarrach and J. P. Deneufville, J. Non-Cryst. Solids 22, 245 (1976).

${ }^{18}$ K. Maruyama, H. Hoshino, H. Ikemoto, and H. Endo, J. Phys. Soc. Jpn. 73, 380 (2004).

${ }^{19}$ J. R. D. Copley, P. Verkerk, A. A. van Well, and H. Fredrikze, Comput. Phys. Commun. 40, 337 (1986).

${ }^{20}$ A. Chiba, Y. Ohmasa, and M. Yao, J. Chem. Phys. 119, 9047 (2003).

${ }^{21}$ C. Cabrillo, F. J. Bermejo, M. Alvarez, P. Verkerk, A. MairaVidal, S. M. Bennington, and D. Martin, Phys. Rev. Lett. 89, 075508 (2002); C. Cabrillo, F. J. Bermejo, A. Maira-Vidal, R. Fernandez-Perea, S. M. Bennington, and D. Martin, J. Phys.: Condens. Matter 16, S309 (2004).

${ }^{22}$ F. J. Bermejo, I. Bustinduy, S. J. Levett, J. W. Taylor, R. Fernandez-Perea, and C. Cabrillo, Phys. Rev. B 72, 104103
(2005).

${ }^{23}$ C. Harikuttan-Unnithan, P. Predeep, S. Jayakumar, and F. Kakinuma, J. Non-Cryst. Solids 351, 754 (2005).

${ }^{24}$ A. Chiba, Y. Ohmasa, Y. Kawakita, M. Yao, and H. Endo, J. Non-Cryst. Solids, 312-314, 384 (2002).

${ }^{25}$ S. W. Lovesey, Theory of Neutron Scattering from Condensed Matter (Oxford Science, Oxford, 1984), Vol. 1, p. 211.

${ }^{26}$ T. Scopigno, R. Di Leonardo, G. Ruocco, A. Q. R. Baron, S. Tsutsui, F. Bossard, and S. N. Yannopoulos, Phys. Rev. Lett. 92, 025503 (2004).

${ }^{27}$ B. Rufflé, M. Foret, E. Courtens, R. Vacher, and G. Monaco, Phys. Rev. Lett. 90, 095502 (2003).

${ }^{28}$ V. Sklyarchuk, Yu. Plevachuk, S. Mudry, and I. Stec, J. Mol. Liq. 120, 111 (2005).

${ }^{29}$ A. B. Bathia and R. N. Singh, Mechanics of Deformable Media (Institute of Physics, Bristol, 1986). 